\title{
Viewpoint
}

\section{Quality health care: the hospital chief executive's role}

\author{
Shelley Heard
}

"Quality goods, quality time, quality health care"-what is this poorly defined but much aspired to characteristic that is used with increasing frequency to describe how we might wish things to be? Why is it that this term, "quality", more perhaps than any other, is being used to shape, identify, and form aspirations around healthcare services? Clinical audit, the patient's charter, and emphasis on clinical effectiveness have been key initiatives in quality healthcare reforms. Most trusts and clinical departments have developed mission statements and objectives which have at their heart an improvement in the quality of care for patients. Although hospital managers are not on the clinical front line, many perceive the strategic and operational context within which they work to be of direct benefit to patients. Certainly those from clinical backgrounds have often made the decision to take up a more strategic role, in which their views on both the direction and quality of health care may influence future outcomes on a more global scale.

The Homerton Hospital was opened in 1986. It was then associated with the world renowned St Bartholomew's Hospital, London's oldest teaching hospital. But in 1994 after the recommendations of a report which considered the shape and organisation of healthcare services in London, it was established as an independent hospital. As its first chief executive I had a clear responsibility to develop local services for the population of Hackney and a unique opportunity to do so within a district general hospital which had as part of its historical ethos a teaching hospital pedigree. The hospital is located in a challenging social and economic environment (box 1). Attitudes about research and teaching were part of the basis of the hospital and it was anticipated from the outset that these would contribute to the creation of a hospital which could combine the best of teaching hospital practice with the development of services responsive to a local community with its own particular problems, needs, and issues.

Box 2 shows the objectives of the chief executive. With the appointment of a management team the process of development began but from the outset the issue of creating quality services was high on the agenda for the chief executive, the management team, and the
- Population of 225000

- 1/4 of population $<15$ years

- Very diverse ethnic population

- Considerable number of refugees $(>25000)$

- Some of the worse indices of socioeconomic deprivation in the United Kingdom

- $<1 / 3$ of adult population have access to private transport

- High morbidity and mortality related to common health complaints-for example, tuberculosis, cancer of the cervix, diabetes

Box 1 Demographic characteristics of Hackney.

clinical and the support staff within the hospital. The only problem was identifying what this elusive term meant and to determine whether it always had the same meaning for all of those who used it.

The quest to develop this understanding was ongoing and resulted in an attempt to engage with all of the stakeholders involved in the delivery of health care at the Homerton -those working in the hospital, the Health Authority and general practitioners which were responsible for purchasing health care for the local population, users, the Community Health

- To achieve the financial obligations of the trust

- To ensure robust, resilient, and timely systems for managing complaints

- To finalise, agree, and implement the trust's quality strategy

- To ensure that standards of the patient's charter are achieved within the constraints of the trust

- To advise the trust board in matters of major strategic importance and to develop for their consideration the five year strategy of the trust

- To develop the trust's annual business plan

- To develop external relations with the Community Health Council, the local authority, the region, voluntary agencies, the press, and the Department of Health

- To develop and support the senior management team to enable them to achieve their corporate and personal goals

- To promote training opportunities for middle managers and for medical staff interested in participating in management

- To introduce a programme of performance management throughout the trust which will encourage and enable staff to meet the corporate objectives of the trust and their individual objectives

Box 2 Objectives of the chief executive. 
Council, and voluntary agencies-to understand how quality healthcare services can be developed for a local community. Reflected in the shared mission statement (box 3) and objectives of the trust, quality was represented on the trust board by the chief executive and a senior manager who had the title of "manager for quality and patient services" and who was directly responsible to the chief executive. The title of the post, although cumbersome, reflected some of the trust's thinking about quality-that it had to do with a close association between clinical audit, patient services, and patients' needs.

From my viewpoint as chief executive continuing strategic attempts to capture the essence of quality services proved to be an uphill battle. Although there was broad agreement by the trust board, senior managers, clinicians, and other stakeholders that quality was about meeting patients' needs, how this was to be translated into reality in a given setting proved to be difficult to determine. We debated within the management team a hospital wide approach and out of it developed a strategic document-Meeting patients' needs: a quality improvement strategy-which encapsulated the developing views of the trust on quality. Its very title signifies that quality is defined by those needs which are important to the patient and not to the clinical staff in the trust. It states that the strategic direction of the trust placed:

"the emphasis and direction of its services upon quality care which is responsive and patient oriented (seeking) to involve staff in the achievement of these aims since the premise of the Trust Board is that staff delivering services are most likely to understand the needs of patients and General Practitioners who access the hospital".

As chief executive, I continued to struggle with clinical staff over this concept of meeting patients' needs, of how to understand what these were in general terms, and what they meant for individual patients. Time and again, through the monitoring of complaints, and through discussions with the Community Health Council and with groups concerned with patients' interests a single theme emerged - given that the clinical care was appropriate and up to professional standards of care, what mattered to the patient was being treated with respect and being given information in a way that was accessible. It required a cultural change within the hospital which would encourage people - both staff and patients - to reconsider their approach to one another, and to find solutions. This could be done within existing systems, which tended to be relatively rigid, or these systems could be abandoned to allow flexibility and tolerance to develop within the constraints of a healthcare system. This does not necessarily promote support for individual patient's needs.

In recognition of this, I developed a draft document entitled $A n A B C$ of patients' expectations (which was eventually called $A n A B C$ of people's expectations, see later, box 4). Its
"The Homerton Hospital NHS Trust aims to offer high quality, reassuring and supportive health care in a responsive environment which promotes health, meets the demands of the community and general practitioners who use it, and supports the staff who work within it.”

Box 3 Mission statement of the Homerton Hospital NHS Trust.

purpose was to engage the whole of the hospital community, general practitioner, groups of patients, and other interested parties in a discussion about the way in which care should be delivered, and to reach a consensus on the values and priorities of the organisation of patients' needs.

Seventeen meetings between the chief executive and different staff groups were held, resulting in a face to face discussion with some 330 members of staff (about $30 \%$ of all the staff) on the values of the people working with the hospital, and the values shared with the local community. Symbolic of the discussions was a suggestion from one member of the portering staff that the $A B C$ should be about peoples' expectations and not just about patients' expectations. This single observation underlined the cultural change that the staff was committed to making and their anticipation that patients would also reflect back attitudes of mutual respect. Eight outside agencies, including two health authorities, two patients' groups, and four general practitioners also responded, supporting the $A B C$ and its principles.

Subsequently, the $A B C$ was adopted by the trust board in a revised form which reflected the consultation process. It has been the cornerstone of the quality strategy for the hospital, and despite concerns that a public statement of such values could hold staff to ransom when things went wrong on the "shop floor", the reality has been that both staff and patients have in full view a statement of shared values and hopes for a quality approach to health care.

In New York City the mayor has announced a "quality of life" programme to improve living within the city. There is little doubt that the combination of an improving economy and a public commitment to improving things is resulting in the city becoming a better place to live. Extreme measures are, however, being used-fines for people "taking up too much space" on the underground, for example, even when there are empty seats! This is clearly one approach to leading from the front. Another is the one which has been adopted by many organisations in the United Kingdom. By setting standards through leadership and through the joint participation of staff, patients, and carers, hospital chief executives have the opportunity to have an important impact on the delivery of quality healthcare. If this is to happen in a serious way, however, then the emphasis on costs, which now seem to be at the core of every discussion on health care, needs to be put into perspective. Margaret O'Kane, the president of the American National Committee for Quality 
Assurance which accredits health maintenance organisations in the United States, has recently said:

"It scares me to think of a system based completely on price. The providers of high quality service will be driven out and the only providers left will be the ones most willing to sacrifice care"

(New York Time Magazine, 8 December 1996).

The acknowledgment that quality care must be a cornerstone of the National Health Service (NHS) has been made clear in the government's recent white paper $A$ service with ambition (London: HMSO, November 1996). The founding principles of the NHS are reiterated in the foreword to the paper-its universality, high quality, and availability on the basis of clinical need. To these the white paper adds a fourth principle - that of responsiveness. The paper relates high quality care to "using the latest knowledge and the highest professional standards", and responsiveness "a service which is sensitive to the needs and wishes of patients and carers". These combined aims need to be translated into grass roots activity within every acute and community unit and within each general practice.

One of the main objectives of chief executives should be to lead their organisations into the development of a culture with quality at its base. Structures which link clinical audit, systems of evidence based decision making, and clinical risk management need to be part of quality initiatives, and may be the outcome measures against which such initiatives should be measured. If, for example, the delivery of an outpatient diabetic service is under review, then one of the aims clearly should be to ensure the quality of that service. If this is an objective then how will its success be assessed? Four criteria should be considered:

(1) Are the protocols within the available evidence and knowledge about the management of diabetes?

(2) Does the practice of the clinic promote the management of clinical risk by providing open and accessible information to patients and their carers?

(3) Is the practice of the clinic supported regularly and consistently by a programme of audit to find what is considered to be best?

(4) Do the structures, systems, and training within the clinic support an ethos of respect and concern for patients or carers as people, above and beyond their clinical needs?

If quality initiatives are to have any real meaning, then there needs to be a clear strategic lead and a well defined means of assessing whether quality objectives have been met. Chief executives need to ensure that the cultures of their organisations reflect this ethos and need to show, through personal commitment and example, that the principles of quality care - professional clinical standards
An $A B C$ of people's expectations at the Homerton Hospital has been developed so that patients, their relatives, and friends who attend the Homerton Hospital, and staff who work within it can begin to share common values about quality health care.

Access to services and help in using them through advocacy and advice

Buildings which are safe and well maintained Confidentiality of all medical and personal details

Dignity for all individuals

Equity in accessing services according to need and clinical priority

Friendly and welcoming staff and environment

Gentle, helpful, and sensitive care

Healthy lifestyle advice in a health promoting environment

Information about the care you can expect and your clinical condition

Joint approach from doctors, nurses, midwives, and therapists in planning care

Kindness and consideration at all times

Listening staff who respond to your questions and concerns

Medical advice and attention when it is required

Needs of the individual recognised

Open, prompt communication with you and your general practitioner

Planning discharge with you, your family, and staff

Quality health care

Respect for your time, for your cultural background, and your social needs

Sympathetic, secure, and responsive care

Trust in the advice you receive about your health Up to date and reliable advice about your health problems

Value and assessment of treatment outcomes

Willingness from the Homerton Hospital and its staff to listen and respond to concerns about your care

Xpert advice from clinical and support staff

Your complaints to be rapidly acknowledged, and thoroughly investigated with appropriate action

Zealous commitment to providing high quality health care

Box $4 A n A B C$ of people's expectations at the Homerton Hospital NHS Trust.

on the one hand and respect and care for the patient on the other-underpin all of clinical practice.

When I took up the post as chief executive at the Homerton my family asked me why I was doing it. I had trained late in medicine, loved seeing patients and being involved in their clinical care, and it was not clear to my family -or even entirely to me-why I would stop "doctoring" to "manage". My response then was that I hoped to establish an organisation which would support the local residents of Hackney, who lived in such difficult social and economic circumstances, and which would put its patients' or users' needs as its primary concern. In my three years as its chief executive I hope that I have achieved this, for in so doing the development of "quality" services will have been assured. 